\title{
Éditorial
}

\section{La revue Organisation \& Territoires et la recherche au DSEA : portrait et perspectives}

La tenue du 86 $6^{\mathrm{e}}$ congrès de l'Association francophone pour le savoir (ACFAS) dans les murs de l'Université du Québec à Chicoutimi (UQAC), en mai 2018, offre à la revue Organisations \& Territoires l'occasion de produire un numéro spécial sur les travaux de recherche effectués au Département des sciences économiques et administratives (DSEA) de l'UQAC, département qui soutient et publie cette revue depuis près de 30 ans.

Durant les années 80, le DSEA publiait P.M.O., la revue de gestion des petites et moyennes organisations, l'ancêtre de la revue actuelle. Puis, à l'automne 1991, paraît le premier numéro d'une version renouvelée et rebaptisée Organisation; la revue est alors axée sur la réflexion et l'intervention en matière de gestion des organisations. Quelques années plus tard, Organisation devient une publication conjointe du DSEA et de l'Institut d'entrepreneuriat de la Faculté d'administration de l'Université de Sherbrooke, en partenariat avec la Fondation de l'Entrepreneurship du Québec et le ministère des Régions.

Un dernier virage important est réalisé à l'automne 1998, alors que la revue adopte son intitulé actuel, Organisations \& Territoires. Conservant son orientation à l'égard des sciences de la gestion, elle entend répondre aux nouvelles exigences imposées aux territoires par l'économie du savoir. Le Centre de recherche sur le développement territorial (CRDT), financé par le Fonds de recherche du Québec - Société et culture (FRQSC), ainsi que le ministère des Affaires municipales et de l'Occupation du territoire (MAMOT) deviennent en 2003 des partenaires à part entière de la revue Organisations \&Territoires et lui apportent un soutien financier annuel.

La revue occupe une place de choix au DSEA, un des plus importants départements de l'UQAC, avec une trentaine de professeurs réguliers et des chargés de cours qui interviennent dans les programmes de premier, deuxième et troisième cycles offerts sur le campus, dans les centres d'études, à l'international, et ce, dans les disciplines suivantes : management, gestion des ressources humaines, économie, finance, marketing, droit et comptabilité.

Afin de mieux faire connaitre la recherche effectuée au DSEA, le comité de la recherche et la direction de la revue Organisations \& Territoires ont voulu produire un numéro spécial multidisciplinaire, mobilisant l'effort des professeurs, des chargés de cours, des étudiants et des professionnels du département. Aussi, ce numéro couvre les divers champs de recherche spécifiques au département, notamment : responsabilité sociale, gouvernance des organisations et développement durable; gestion des organisations et gestion de projet; marketing stratégique et comportement humain; comptabilité et fiscalité; santé, sécurité et bien-être au travail. Enfin, il importe de souligner que de nombreux professeurs réalisent leurs travaux de recherche au sein du CRDT ou des divers laboratoires de recherche et d'intervention. 
Ce numéro spécial publié à l'occasion de la tenue de l'ACFAS permet à la revue d'adopter un nouveau visage; son image sera dorénavant mise en valeur par une nouvelle maquette à chaque publication, reflétant l'effervescence de la recherche et de l'intervention en sciences de la gestion. L'autonomie réflexive du gestionnaire requiert l'assimilation de savoirs produits par diverses disciplines ou professions. En développant sa compétence face à la complexité, le gestionnaire entreprend de mieux participer à l'animation des changements visant les nombreux processus globalisés de la planète et leurs problématiques inédites.

En plus de ses vingt-et-un articles, ce numéro comporte deux rubriques : la première, sur les " unités de recherche », présente les travaux en cours, les orientations de recherche et leurs membres; la seconde fait connaitre les projets de thèse des étudiants au doctorat en management de projets (DMP).

On comprendra que c'est avec beaucoup de fierté que nous publions cette édition de la revue Organisations \& Territoires. Nous espérons que l'on aura autant de plaisir à sa lecture que nous en avons eu à la produire. Ce fut un privilège de mobiliser autant de professeurs, de chargés de cours, d'étudiants et de personnels sur la recherche en cours au DSEA. Rendez-vous à l'ACFAS au printemps 2018 !

Thierno Diallo

Directeur de la revue Organisations \& Territoires

Jeanne Simard

Professeure et coordonnatrice du numéro spécial DSEA-ACFAS 